\title{
A Check List to Reduce Misuse of Primary Cesarean Sections in Women with a Single Fetus in Cephalic Position
}

\author{
Catalina Acuña Pradilla1, Delgzar Raúl Gómez Pereira1, Luis Alfonso Díaz-Martínez ${ }^{2 *}$ \\ ${ }^{1}$ Gyneco Obstetrics Department, School of Medicine, Faculty of Health, Universidad Industrial de Santander, Bucaramanga, Colombia \\ ${ }^{2}$ School of Medicine, Faculty of Health, Universidad Industrial de Santander, Bucaramanga, Colombia \\ Email: *ladimar@uis.edu.co
}

How to cite this paper: Pradilla, C.A., Pereira, D.R.G. and Díaz-Martínez, L.A. (2017) A Check List to Reduce Misuse of Primary Cesarean Sections in Women with a Single Fetus in Cephalic Position. Health, 9, 1251-1263.

https://doi.org/10.4236/health.2017.98090

Received: May 27, 2017

Accepted: August 27, 2017

Published: August 30, 2017

Copyright $\odot 2017$ by authors and Scientific Research Publishing Inc. This work is licensed under the Creative Commons Attribution International License (CC BY 4.0).

http://creativecommons.org/licenses/by/4.0/

\begin{abstract}
Background: Avoiding primary C-section is the safest and most effective way of decreasing $\mathrm{C}$-section rates. We analyzed circumstances and decisions made among pregnant women without history of $\mathrm{C}$-section and cephalic single fetus (group B), who ended up having a Cesarean birth, to identify opportunities that may optimize the decisions about delivery. Methodology: We evaluated the clinical histories of pregnant women from group B who completed their pregnancies in the Hospital Universitario de Santander (Bucaramanga, Colombia) during 2013. We evaluated the decision moments from admission until birth, including the registry of the reasons for having a C-section, compliance of all the criteria to support the decision and its appropriateness, and how adequate were the procedures done during the induction or augmentation of labor. Results: We evaluated 1320 histories; 666 (55.7\%) ended by C-Section. In $59.8 \%$ of the histories, we identified at least one decision that could have been addressed differently to optimize the delivery type and, potentially, avoid ending in a Cesarean birth. The most frequent opportunities arise due to a lack of clarity in the record of the reason for performing the C-section (70.1\%), and inadequate use of labor induction in the patients that had to end their gestation (38.5\%). Conclusion: In the Hospital Universitario de Santander during 2013, despite observing a meaningful subregistry of the indication to perform a C-section, we found that in one out of every two patients there are opportunities of improvement in the registry and attention to reduce the high incidence of $\mathrm{C}$-sections in the institution. This analysis allowed us to create a checklist to fill out before making the final decision of performing a Cesarean birth.
\end{abstract}

\section{Keywords}

Labor, Delivery Type, Cesarean Birth 


\section{Introduction}

C-sections are procedures that save lives, both of mothers and fetuses. Since its popularization as a tool to diminish maternal and perinatal mortality, the rate of Cesarean births has been increasing, until being the first indication for major surgery in developed countries [1]. Currently, 18.6\% of global births are C-sections, ranging from $6 \%$ in the less developed regions, to $27.2 \%$ in the more developed ones; in Latin America and the Caribbean, the highest rates for Cesarean births are evidenced [2], and specifically in Colombia, $45 \%$ of births are C-sections [3]. As in every surgical procedure, a C-section implies maternal and perinatal risks, both immediate and long term. These risks should be considered in the cases in which vaginal birth poses a threat to the health of the mother or baby, given the fact that it is not an innocuous intervention [4].

The World Health Organization (WHO) states that many Cesarean births in the world are not fully justified and that maternal or fetal complications derived from it could be interpreted as iatrogenic [5]. In spite of the fact that the rate of births that end up in a C-section keeps increasing, WHO establishes that it is not possible to propose a universal institutional figure of reference for this rate, because it depends on the complexity of present maternal and fetal conditions, the characteristics of the population cared for, and the available resources [6]. These elements play a fundamental role when making a decision about performing a C-section or not. It also states that, among other things, a Cesarean should be performed when it is medically necessary and that, instead of exerting to obtain a given Cesarean rate, the initiatives should focus on guaranteeing its performance on pregnant women who need it, without abusing or corrupting the reasons to indicate it [6].

In an effort to diminish the rate of $\mathrm{C}$-sections, multiple strategies have been established. Two of them are the trial of labor after a Cesarean or the vaginal delivery of multiple pregnancies, which have not had the expected success due to the fear to associated maternal and fetal complications, and also to the potential medico-legal risks that an adverse outcome could imply [7]. Recently, the efforts have focused on reducing the incidence of primary C-section, meaning avoiding the procedure in pregnant women who have never had one, unless there was a fetal or maternal condition that would make it necessary. From this perspective, and taking into account the fact that there are very few absolute indications for a C-section (for example, placenta previa with total occlusion), the other reasons for indicating a primary Cesarean depend on the interpretation and the response of the health team to clinical situations of the pregnancy or labor [8].

Due to the fact that the C-section is a medical action that has to be supported, it is particularly important to clearly establish the reasons to perform it on pregnant women with cephalic single fetus and no history of uterine scarring, as they should end their gestation by vaginal birth, unless a clear indication exists. The reasons to perform a C-section on women with a high probability of ending their pregnancy by Cesarean (multiple pregnancy, non-cephalic presentation, previous 
C-section), are easier to accept, while among other patients solid argumentation is needed to validate such a decision. This leads to the possibility of identifying the former women as patients from group A, since the probability of a C-section is high, while identifying the latter as group $B$, where the Cesarean decision must be sufficiently supported. Thus, the population from group B would be more susceptible to the modifications in the medical or administrative procedures involved in decision-making about delivery type and, as such, they are the object of efforts to provide adequate care without resorting to unjustified Cesareans. Therefore, every decision moment, from the admission of the patient to a health service until birth, could be considered as an opportunity to optimize the $\mathrm{C}$-section rate of that institution.

This article presents the analysis of the circumstances and the decisions made among pregnant women from group B in a Colombian institution, emphasizing those that ended their pregnancy by $\mathrm{C}$-section, to determine the type and frequency of improvement opportunities that may be suggested to improve the process and opportunity of decision-making that lead to perform a Cesarean according to the conditions of the patients at the institution.

\section{Methodology}

A descriptive study of the clinical histories of pregnant women that ended gestation in the Hospital Universitario de Santander (Bucaramanga, Colombia) during 2013 was carried out. This is a third level public university hospital and a reference center for the obstetric population in the Colombian northeast. The study was approved by the Review Board of the Universidad Industrial de Santander, without the need of a written consent due to the fact that the study was based on the evaluation of the clinical charts.

From the 1818 pregnant women that gave birth at the institution, we identified that 1320 were from group B (single pregnancy, cephalic presentation and no history of uterine scarring due to C-section or myomectomy). From these histories, we systematically extracted information using a predesigned guide with sociodemographic variables (age and origin); if she had been referred or not to Hospital Universitario de Santander from another institution; obstetric formula, gestational age at the moment of birth and the way labor started (spontaneous, induced or Cesarean without start of labor); delivery type (vaginal delivery or C-section), and the two main reasons to indicate the C-section, in case there were more than one. All the information was typed in duplicate in Microsoft $^{\circledR}$ Excel $^{\circledR}$.

All the histories were analyzed by two authors (DRGP and CAP); one of them is a graduate obstetrician and gynecologist and graduate program professor, who works directly in the delivery room, and the other one is a last year student from the obstetrics and gynecology graduate program.

The evaluation of each history was done using another guiding tool to establish the following by consensus: a) the main indications to perform the C-section 
among those stated; b) the pertinence of the indications to perform the Cesarean according to the evidence in the clinical history; $c$ ) the adherence to internationally accepted guidelines at the moment of delivery related to the decision of performing the C-section [8]-[13].

Finally, the objective was to identify the institutional opportunities to improve the care offered to the patients, aiming at the reduction of needed C-sections. This focus generated several categories that were based on Spong et al. [8] and according to our interpretation on the reasons to do each $\mathrm{C}$-section as well as on the adherence to the care protocols according to the clinical condition of each patient [9] [10] [11] [12] [13]; by this way, the outlined categories were:

- The existence or not of one or more reasons that justified the Cesarean.

- Those referring to the diagnosis that justifies the $\mathrm{C}$-section to the extent that: a) the clinical or paraclinical criteria that support the given diagnosis exist; b) the fact that there is a diagnosis is not necessarily a valid criteria on its own for the performance of a C-section if a condition of risk for the mother or child during the birth is not reflected on the history (for example, premature birth C-section without evidence of fetal well-being alteration); or c) the registered information was not coherent.

- Those referring to the labor induction: if it was not performed when indicated; or if it was not performed correctly before the obstetric situation progressed to the point of counter-indicating vaginal birth; or if the actions or times during the induction were inadequate.

- Those referring to the monitoring and management of the labor in the cases where it was not counter-indicated: labor was not performed or spontaneous progress was not allowed.

- The existence of a valid indication to end gestation previous to the start of spontaneous labor.

- The performance of uterine perfusion maneuvers (only for those patients with an unsatisfactory fetal status diagnosis).

- The existence of a medical indication that is not considered as valid to perform a C-section, including the mother's request isolated from the patient's clinical condition.

The statistical analysis was done in Stata 12.1 (StataCorp, College Station, EU, 2014). Demographical and clinical differences were established through proportions or measures of central tendency and dispersion. The main C-section indications and the opportunities were presented as a proportion, and the opportunities were classified into several categories as was previously presented, with the possibility of a patient having more than one opportunity to improve the decision-making related to delivery type.

\section{Results}

The age of the 1320 pregnant women from group B who ended their gestation during 2013 in the Hospital Universitario de Santander ranged between 13 and 
48 years old ( 22 years mean, IQR 19 to 28); the gestational age ranged between 22 and 42 weeks (38 weeks mean, IQR 36 to 39); 348 (26.4\%) were preterm; 726 (55.0\%) were nulliparous (without any vaginal delivery history) and 845 (64.3\%) were in their first pregnancy; 684 (51.8\%) were referrals from other centers and $154(11.7 \%)$ without an adequate prenatal care. A total of $666(55.7 \%)$ ended their pregnancies by C-section, 379 (56.9\%) did not start labor, 84 (12.6\%) after its induction, and 203 (30.5\%) after spontaneous start of labor (Figure 1); the median birthweight was 2940 gr (IQR 2470-3330).

The analysis of the 666 histories of the patients from group B that had a C-section shows that more than half of the patients had a Cesarean due to a non-satisfactory fetal status, hypertensive disorders or labor dystocias as the main indication for the procedure (Table 1). In 345 patients (51.8\%), opportunities to optimize the decision-making about delivery type were found, either at the moment of diagnosis or during management. These opportunities occur more frequently with diagnoses related to failed induction, labor dystocia and non-satisfactory fetal status, apart from those derived from the patients without a clear medical indication.

In Table 2, seven opportunity groups to optimize the decision-making in order to perform a C-section are shown. The most frequent opportunity group is the one related to failures in the clinical history (239 patients, $70.1 \%$ ). Although in $104(30.5 \%)$ of them, a clear justification was not found, be it valid or not, in $87(83.7 \%)$ it was possible to establish the potential reason to perform a C-section given the clinical context that the history revision allowed us to determine.

The second opportunity group was made up by 133 (38.6\%) patients in which the labor induction process had failures, and the third one was due to failures in the registered diagnosis to perform the C-section (118 patients, 34.2\%). Among the 133 patients that had a Cesarean due to non-satisfactory fetal status, intra-uterine resuscitation was not conducted in 77 (57.9\%) patients.

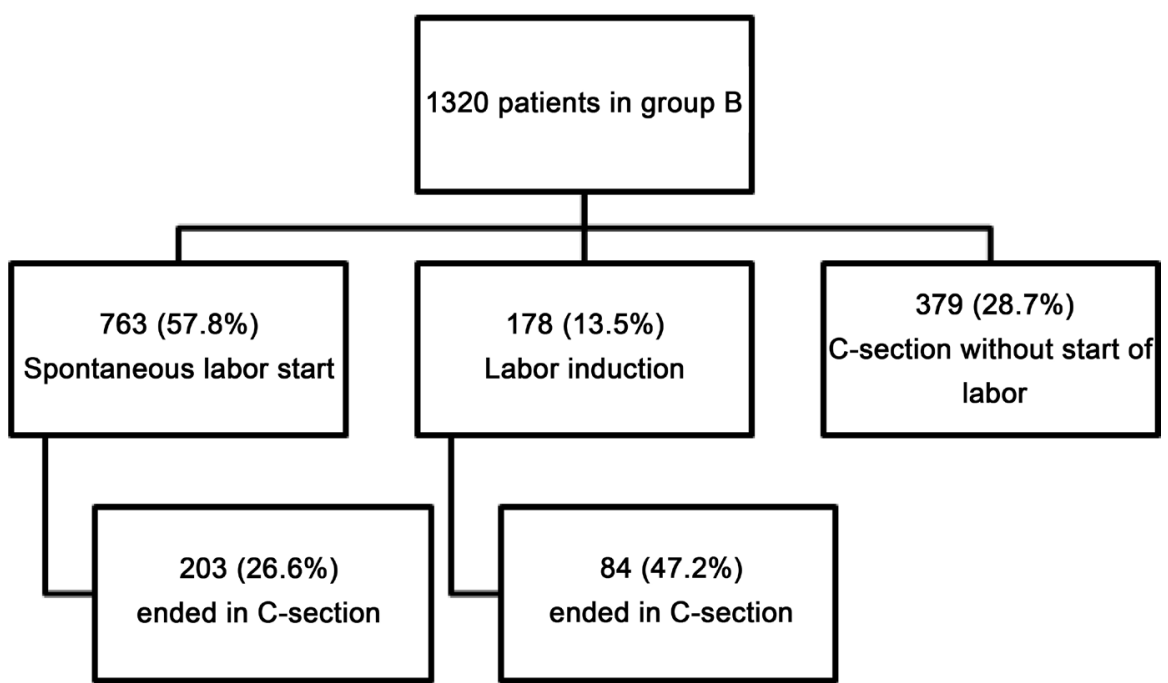

Figure 1. Way in which the patients from group B started their labor and how they finished it. 
Table 1. Indication for C-section in group B and found opportunities according to the primary indication.

\begin{tabular}{|c|c|c|}
\hline Indication for primary C-section & n (\%) & $\begin{array}{l}\text { Patients with identified improvement } \\
\text { opportunities (\% over the total of the } \\
\text { group) }\end{array}$ \\
\hline Unsatisfactory fetal state & $133(19.9 \%)$ & $80(60.2 \%)$ \\
\hline Hypertensive disorders & $131(19.6 \%)$ & $29(22.2 \%)$ \\
\hline Labor dystocias & $121(18.1 \%)$ & $99(81.8 \%)$ \\
\hline IUGR & $71(10.6 \%)$ & $22(31.0 \%)$ \\
\hline No medical indication & $43(6.6 \%)$ & $43(100 \%)$ \\
\hline Amniotic fluid alterations & $42(6.3 \%)$ & $11(26.1 \%)$ \\
\hline Macrosomia & $42(6.3 \%)$ & $18(42.8 \%)$ \\
\hline Failed induction & $26(3.9 \%)$ & $25(96.2 \%)$ \\
\hline Placental pathologies & $18(2.7 \%)$ & - \\
\hline Chorioamnionitis & $9(1.4 \%)$ & $7(77.8 \%)$ \\
\hline Extreme pretermbirth & $5(0.8 \%)$ & $5(100 \%)$ \\
\hline Other & $25(3.6 \%)$ & $6(25.0 \%)$ \\
\hline Total patients & $666(100 \%)$ & $345(51.8 \%)$ \\
\hline
\end{tabular}

Table 2. Opportunities to optimize the C-section decision.

\begin{tabular}{|c|c|c|}
\hline Group and type of opportunity & Patients & $\begin{array}{l}\text { Proportion } \\
(\mathrm{n}=345)\end{array}$ \\
\hline \multicolumn{3}{|l|}{ Failure in the clinical history } \\
\hline No clarity in the justification & 104 & $30.5 \%$ \\
\hline No information necessary for the diagnosis & 91 & $26.7 \%$ \\
\hline Lack of the key paraclinic for the diagnosis & 25 & $7.3 \%$ \\
\hline Non concordant information & 19 & $5.6 \%$ \\
\hline \multicolumn{3}{|l|}{ Failure in labor induction } \\
\hline Induction was not performed & 84 & $24.6 \%$ \\
\hline Timely induction was not performed & 22 & $6.5 \%$ \\
\hline Inadequate induction & 27 & $7.9 \%$ \\
\hline \multicolumn{3}{|l|}{ Failure in the registered diagnosis that led to the C-section } \\
\hline Diagnosis criteria are not met & 85 & $24.9 \%$ \\
\hline Diagnosis is not a criterion on its own & 33 & $9.7 \%$ \\
\hline Labor was not conducted & 82 & $24.1 \%$ \\
\hline In uterus reanimation was not done & 77 & $22.6 \%$ \\
\hline No medical indication to perform a C-section & 43 & $12.6 \%$ \\
\hline No indication to end gestation before the start of labor & 32 & $9.4 \%$ \\
\hline
\end{tabular}




\section{Discussion}

Avoiding primary C-section is the most effective way to decrease Cesarean rates [8]. In the Hospital Universitario de Santander, $55.7 \%$ of the patients without uterine scarring history and with a cephalic single fetus ended their gestation by C-section; in more than half of them, we found at least one moment during hospital care that could have been dealt with differently, which turns them into opportunities to improve the delivery service process, and also, to reduce C-section rates. The main opportunities to improve detected in the Hospital Universitario de Santander are the lack of clarity registered in the clinical history about the reason to perform the $\mathrm{C}$-section and the inadequate use of labor induction among those patients that had to end their gestation due to medical indications.

This study is retrolective, based on what exists in the clinical histories; it is possible that the problem mentioned above is more of a lack of registration of the information, than neglect or mistakes during hospital care (for example, patients without cervical change progression that is not registered in the partograph). However, given the fact that the clinical history is a legal document, the lack of registration of medical acts and the supports they are based on are in themselves a failure in health care [8].

This study was done using all the population cared for at the Hospital Universitario de Santander during 2013, reason why there is no selection bias; additionally, the histories were evaluated by two obstetricians who made the decisions by consensus and based on the accepted protocols at the time of service to the patients for each type of clinical situation [8]-[13]. However, the conformation of the clinical group of evaluators could be considered as a limitation of the study to the extent that the analysis shown here has to be done by personnel who are not involved in the care of these patients. Despite that, to reduce this potential bias to the maximum, we defined the variables and the analysis strategies $a$ priori, including choosing the clinical attention guidelines used at the Hospital Universitario de Santander.

Birth on its own, regardless of its type, carries risks for the mother and the child. We can also add to those the risk of maternal or fetal morbidity when performing a C-section, and those inherent to the surgical procedure itself [14]. For example, a Cesarean increases the risk of maternal death threefold [15] or neonatal breathing difficulty, which can go from less than $<1 \%$ up to $4 \%$ [16]. Also, a C-section increases the risk of complications in future gestations (for example, abnormalities of the placental insertion or uterine rupture), which can also lead to an increase in the need of new C-sections and secondary perinatal morbidity to a major potential for the need of emergency or elective Cesarean without labor [17]. With it being a procedure which marks the reproductive future, each patient taken to a primary $\mathrm{C}$-section must be given the explanation about the long term implications of the procedure and its indication, making sure she understands, stating everything clearly in the informed consent and the clinical history [8]. 
The decision of ending a gestation that has not started spontaneous labor must be based on the assessment of the risk of continuing gestation over induction risks or those of a subsequent C-section in case that the induction fails [8]. The inductions performed for the patient's or doctor's comfort (for example, mobility issues by living in a rural area) increase the possibility of ending in a C-section compared to those patients that start labor spontaneously [14]. In this study, improvement opportunities were found in $9.4 \%$ of the patients, who could have continued with their gestation until the spontaneous start of labor due to the fact that they did not had a valid indication to end it at that time.

When it is established that a gestation must end before the spontaneous start of labor, the first alternative is to consider inducing it in order for it to end by vaginal delivery, unless there is a contraindication [8]. The probability of achieving a vaginal birth during the induction is lower to the one given after the spontaneous start of labor, mainly in nulliparous patients with unfavorable cervix [18]. There is controversy in terms of the ideal method to perform a cervical maturation [19], but in any case, it should be attempted if necessary and the institutional protocols must standardize the use of cervical maturation methods and oxytocin in order to be able to evaluate their success in each patient and the effectiveness of the protocol itself [14]. Thus, up to $7.9 \%$ of the evaluated patients in this study could have benefited from the adherence to such protocols.

Spontaneous start of labor is an ideal situation for a pregnant woman to end her pregnancy [8]. The times of each phase of labor must be respected, provided that the maternal and fetal conditions allow it [14]. It is considered that a C-section is justified due to labor stall when there is a pregnant woman with a 6 $\mathrm{cm}$ dilatation and with ruptured membranes one of the two following conditions is given: a) no evidence of labor progression even after regular uterine activity for four hours or b) despite at least six hours support with oxytocin to conduct labor, without accomplishing an adequate uterine dynamic or cervical changes [20]. On the other hand, the cephalopelvic disproportion diagnosis goes beyond a mere assessment of the maternal pelvis and it should include trial of labor to dynamically evaluate its progression [8]. According to this, in this research up to $24 \%$ of the total improvement opportunities occur due to non-adherence to labor conduction protocols [8]-[13].

The most used method to assess the fetus' well being, with or without labor, is fetal monitoring [12]. However, its systematic use has not turned into a reduction of cerebral palsy cases, but into an increase in C-section rates; there is also, no greater benefit with continuous monitoring of the fetal heart rate versus intermittent auscultation [21]. It seems that the issue is more with monitoring protocols and adequate assessment of the findings with one method or the other. For example, most tracings during birth are category II; in other words, they are indeterminate and imply having constant monitoring, evaluating the possible causes and establishing the needed corrective measures [22]. Category III monitoring frequency is occasional and almost always implies an emergency C-section 
[12]; these category II tracings are used to justify many Cesareans [14]. For this reason, it is fundamental to make sure that the patients with these tracings have the adequate clinical measures in order to solve the circumstances that lead to the unspecified alterations that they represent, aiming to ensure fetal well-being and without rushing a C-section in order to solve the situation [12]. One simple strategy is the fetal head stimulation, which is expected to cause fetal heart rate acceleration, which indicates the fetus' well-being [23]. The use of uterine perfusion measures is a common component for handling abnormal tracings; nevertheless, the data are too limited in terms of their true usefulness [14]. Up to $22 \%$ of improvement opportunities in the Hospital Universitario de Santander are related to the optimization of the adherence to uterus reanimation protocols; this group of patients could have benefited from this maneuver, either to continue labor or to improve the acid-base status of the newborn.

In the Hospital Universitario de Santander, maternal or fetal morbidity are $47.7 \%$ of the main reasons to perform a C-section in patients from group B, much higher to what has been informed in general populations [24]. Nevertheless, to adequately compare this information, it is necessary to consider the risk structure of the pregnant women population cared for at each center [25]; for our figures, really there are not other reports to compare because the definition of group B has been generated for this report. In these sense, the indirectstandadized C-section rate for other Centers were $43.1 \%$ at Hospital Universitario San Vicente Fundación, Colombia, 2011-2012 [26], 46.6\% at Women's Hospital at the University of Campinas, Brazil, 2009-2013 [27], 16.1\% at Hospital La Inmaculada, Spain, 2006-2013 [28], 42.9\% at Gemelli University Hospital, Italy, 2010-2011 [29], or 27.4\% at Canada, 2010-2011 [30].

The majority of patients at the Hospital Universitario de Santander were taken to a C-section without labor or induction, with them being $56.9 \%$ of the Cesareans from group B. The vast majority of maternal or fetal pathological conditions are not an absolute indication for a C-section, and most of them do not worsen after a trial of labor: for example, preeclampsia or intrauterine growing restriction with a category III Doppler [31] [32] [33].

Besides the improvement opportunities detected in this exercise, there are other equally necessary measures that have also shown their usefulness: changing the way in which health professionals and the general public perceive labor as something dangerous that makes both the mother and child suffer [8], a C-section as an innocuous procedure [4], the fear of legal repercussions from any apparently adverse outcome [34], and the type of economical or work benefit obtained by a C-section or vaginal birth, for professionals, institutions and health service payers [8] [35].

There are also institutional measures that might help to optimize the use of this resource: labor assistance with labor pain reduction methods [3]; the presence of a companion during birth [36], the measure of a double assessor at the moment of indicating the $\mathrm{C}$-section, or the permanent evaluation of the clinical 
Table 3. Checklist to optimize decision-making about delivery type. Hospital Universitario de Santander, Colombia, 2016. Doctor. If you have not filled the twelve conditions or at least one of your answers is "No", reassess the need for a C-section because it is not fully justified.

\begin{tabular}{lll}
\hline Aspects to evaluate & Yes No N/A
\end{tabular}

Allowing a normal progress of labor is contraindicated

There is an indication to end gestation before the spontaneous start of labor

Labor induction or augmentation are contraindicated

Induction or augmentation of labor were performed

Induction or augmentation was adequate

There is an accepted medical indication for the performance of a C-section

There is compliance with all diagnostic criteria of the indication for C-section

All clinical and paraclinical information necessary for the diagnosis that

indicates performing the $\mathrm{C}$-section is available

The information in the clinical history is concordant and complete

The justification for the C-section is clearly stated in the clinical history

In patients with unsatisfactory fetal state, the possible causes that could explain

the alterations in fetal well-being were evaluated and corrected

In patients with unsatisfactory fetal state, uterine perfusion maneuvers were performed

N/A: Not apply.

histories of the patients taken to a Cesarean, especially those with an unsatisfactory fetal state indication, labor stall or failed induction [8]. As a matter of fact, from the analysis of the 666 patients from group B that ended their pregnancy by Cesarean birth in 2013 in the Hospital Universitario de Santander, we developed a checklist to be filled out before deciding on a C-section, derived from the opportunities shown here (Table 3 ). This list seeks to guarantee that decision-making processes to choose a $\mathrm{C}$-section are systematically considered in all patients in a way in which all performed Cesareans are fully justified.

\section{References}

[1] Boyle, A., Reddy, U.M., Landy, H.J., Huang, C.-C., Driggers, R.W. and Laughon, S.K. (2013) Primary Cesarean Delivery in the United States. Obstetrics \& Gynecology, 122, 33-40. https://doi.org/10.1097/AOG.0b013e3182952242

[2] Betrán, A.P., Ye, J., Moller, A.-B., Zhang, J., Guilmezoglu, A.M. and Torloni, M.R. (2016) The Increasing Trend in Caesarean Section Rates: Global, Regional and $\mathrm{Na}$ tional Estimates: 1990-2014. PLoS ONE, 11, e0148343.

[3] Rubio-Romero, J.A., Fonseca-Pérez, J.E., Molina, S., Sánchez, A., et al. (2014) Colombian Obstetrics and Gynecology Federation and Colombian Perinatology Federation Consensus. Rationalization of C-Section Usage in Colombia, 2014. Revista Colombiana de Obstetricia y Ginecología, 65, 139-151. (In Spanish)

[4] Souza, J.P., Gülmezoglu, A., Lumbiganon, P., Lappaiboon, M., Carroli, G., Fawole, B., et al. (2010) Caesarean Section without Medical Indications Is Associated with 
an Increased Risk of Adverse Short-Term Maerna Outcomes: The 2004-2008 WHO Global Survey on Maternal and Perinatal Health. BMC Medicine, 8, 71.

https://doi.org/10.1186/1741-7015-8-71

[5] Villar, J., Valladares, E., Wojdyla, D., Zavaleta, N., Carroli, G., Velazco, A., et al. (2006) Caesarean Delivery Rates and Pregnancy Outcomes: The 2005 WHO Global Survey on Maternal and Perinatal Health in Latin America. Lancet, 367, 1819-1829. https://doi.org/10.1016/S0140-6736(06)68704-7

[6] Betran, A.P., Torloni, M.R., Zhang, J.J. and Gülmezoglu, A.M. (2016) WHO Working Group on Caesarean Section. WHO Statement on Caesarean Section Rates. British Journal of Obstetrics and Gynaecology, 123, 667-670. https://doi.org/10.1111/1471-0528.13526

[7] American College of Obstetricians and Gynecologists (2010) Practice Bulletin No. 115: Vaginal Birth after Previous Cesarean Delivery. Obstetrics \& Gynecology, 116, 450-463. https://doi.org/10.1097/AOG.0b013e3181eeb251

[8] Spong, C.Y., Berghella, V., Wenstrom, K.D., Mercer, B.M. and Saade, G.R. (2012) Preventing the First Cesarean Delivery: Summary of a Joint Eunice Kennedy Shriver National Institute of Child Health and Human Development, Society for Maternal-Fetal Medicine, and American College of Obstetricians and Gynecologists Workshop. Obstetrics \& Gynecology, 120, 1181-1193.

[9] American College of Obstetricians and Gynecologists (2013) Practice Bulletin No. 134: Fetal Growth Restriction. Obstetrics \& Gynecology, 121, 1122-1133. https://doi.org/10.1097/01.AOG.0000429658.85846.f9

[10] American College of Obstetricians and Gynecologists (2002) Practice Bulletin No. 33: Diagnosis and Management of Preeclampsia and Eclampsia. Obstetrics \& Gynecology, 99, 159-167.

[11] Eixcharh, E. and Figueras, F. (2014) Oligohydramniosin Single Gestation. Clinical Guide. Hospital Clínic de Barcelona, Barcelona. (In Spanish)

[12] American College of Obstetricians and Gynecologists (2010) Practice Bulletin No. 116: Management of Intrapartum Fetal Heart Rate Tracings. Obstetrics \& Gynecology, 116, 1232-1240. https://doi.org/10.1097/AOG.0b013e3182004fa9

[13] Rossi, C., Mullin, P. and Prefumo, F. (2013) Prevention, Management, and Outcomes of Macrosomia: A Systematic Review of Literature and Meta-Analysis. $O b$ stetrical and Gynecological Survey, 68, 702-709. https://doi.org/10.1097/01.ogx.0000435370.74455.a8

[14] American College of Obstetricians and Gynecologists (College); Society for Maternal-Fetal Medicine, Caughey, A.B., Cahill, A.G., Guise, J.M. and Rouse, D.J. (2014) Safe Prevention of the Primary Cesarean Delivery. American Journal of Obstetrics \& Gynecology, 210, 179-193. https://doi.org/10.1016/j.ajog.2014.01.026

[15] Liu, S., Liston, R.M., Joseph, K.S., Heaman, M., Sauve, R. and Kramer, M.S. (2007) Maternal Mortality and Severe Morbidity Associated with Low-Risk Planned Cesarean Delivery versus Planned Vaginal Delivery at Term. Maternal Health Study Group of the Canadian Perinatal Surveillance System. CMAJ, 176, 455-460. https://doi.org/10.1503/cmaj.060870

[16] Gregory, K.D., Jackson, S., Korst, L. and Fridman, M. (2012) Cesarean versus Vaginal Delivery: Whose Risks? Whose Benefits? American Journal of Perinatology, 29, 7-18. https://doi.org/10.1055/s-0031-1285829

[17] Miller, E.S., Hahn, K. and Grobman, W., for the Society for Maternal-Fetal Medicine Health Policy Committee (2013) Consequences of a Primary Elective Cesarean Delivery across the Reproductive Life. Obstetrics \& Gynecology, 121, 789-797. https://doi.org/10.1097/AOG.0b013e3182878b43 
[18] Stock, S.J., Ferguson, E., Duffy, A., Ford, I., Chalmers, J. and Norman, J.E. (2012) Outcomes of Elective Induction of Labour Compared with Expectant Management: Population Based Study. BMJ, 344, e2838. https://doi.org/10.1097/01.ogx.0000421444.97113.83

[19] World Health Organization (2011) WHO Recommendations for Induction of Labour. WHO, Geneva.

[20] Zhang, J., Landy, H.J., Branch, W., Burkman, R., Haberman, S., Gregory, K.D., et al. (2010) Contemporary Patterns of Spontaneous Labor with Normal Neonatal Outcomes. Obstetrics \& Gynecology, 116, 1281-1287. https://doi.org/10.1097/AOG.0b013e3181fdef6e

[21] Sameshima, H., Ikenoue, T., Ikeda, T., Kamitomo, M. and Ibara, S. (2004) Unselected Low-Risk Pregnancies and the Effect of Continuous Intrapartum Fetal Heart Rate Monitoring on Umbilical Blood Gases and Cerebral Palsy. American Journal of Obstetrics \& Gynecology, 190, 118-123. https://doi.org/10.1016/j.ajog.2003.07.014

[22] American College of Obstetricians and Gynecologists (2009) Practice Bulletin No. 106: Intrapartum Fetal Heart Rate Monitoring: Nomenclature, Interpretation, and General Management Principles. Obstetrics \& Gynecology, 114, 192-202. https://doi.org/10.1097/AOG.0b013e3181aef106

[23] Elimian, A., Figueroa, R. and Tejani, N. (1997) Intrapartum Assessment of Fetal Well-Being: A Comparison of Scalp Stimulation with Scalp Blood pH Sampling. Obstetrics \& Gynecology, 89, 373-376. https://doi.org/10.1016/S0029-7844(96)00525-X

[24] Barber, E.L., Lundsberg, L.S., Belanger, K., Pettker, C.M., Funai, E.F. and Illuzzi, J.L. (2011) Indications Contributing to the Increasing Cesarean Delivery Rate. Obstetrics \& Gynecology, 118, 29-38. https://doi.org/10.1097/AOG.0b013e31821e5f65

[25] Robson, M.S. (2001) Classification of Caesarean Sections. Fetal and Maternal Medicine Review, 12, 23-39. https://doi.org/10.1017/S0965539501000122

[26] Zuleta, J.J., Quintero, F. and Quiceno, A. (2013) Application of the Robson Model to Characterize the Relation of C-Sections in a Third Level Care Institution in Medellin, Colombia. A Cross-Sectional Study. Revista Colombiana de Obstetricia y Ginecología, 64, 90-99. [In Spanish]

[27] Ferreira, E.C., Pacagnella, R.C., Costa, M.L. and Cecatti, J.G. (2015) The Robson ten-Group Classification System for Appraising Deliveries at a Tertiary Referral Hospital in Brazil. International Journal of Gynecology \& Obstetrics, 219, 236-239. https://doi.org/10.1016/j.ijgo.2014.11.026

[28] Aguilar, R., Manrique, G., Aisa, L.M., Martínez, L., González, V. and Aceituno, L. (2016) Use of Robson's Classification in a Regional Hospital in Spain to Reduce the Rate of Cesarean Sections. Revista Chilena de Obstetricia y Ginecologia, 81, 99-104. [In Spanish]

[29] Triunfo, S., Ferrazzani, S., Lanzone, A. and Scambia, G. (2015) Identification of Obstetric Targets for Reducing Cesarean Section Rate Using the Robson Ten Group Classification in a Tertiary Level Hospital. European Journal of Obstetrics \& Gynecology and Reproductive Biology, 189, 91-95. https://doi.org/10.1016/j.ejogrb.2015.03.030

[30] Kelly, S., Sprague, A., Fell, D.B., Murphy, P., Aelicks, N., Guo, Y., et al. (2013) Examining Caesarean Section Rates in Canada Using the Robson Classification System. Journal of Obstetrics and Gynaecology Canada, 35, 206-214. https://doi.org/10.1016/S1701-2163(15)30992-0 
[31] Figueras, F. and Gratacós, E. (2014) Update on the Diagnosis and Classification of Fetal Growth Restriction and Proposal of a Stage-Based Management Protocol. Fetal Diagnosis and Therapy, 36, 86-98. https://doi.org/10.1159/000357592

[32] Coppage, K.H. and Polzin, W.J. (2002) Severe Preeclampsia and Delivery Outcomes: Is Immediate Cesarean Delivery Beneficial? American Journal of Obstetrics \& Gynecology, 186, 921-923. https://doi.org/10.1067/mob.2002.124041

[33] Alanis, M.C., Robinson, C.J., Hulsey, T.C., Ebeling, M. and Johnson, D.D. (2008) Early-Onset Severe Preeclampsia: Induction of Labor vs Elective Cesarean Delivery and Neonatal Outcomes. American Journal of Obstetrics \& Gynecology, 199, e1-e6. https://doi.org/10.1016/j.ajog.2008.06.076

[34] Yang, Y.T., Mello, M.M., Subramanian, S.V. and Studdert, D.M. (2009) Relationship between Malpractice Litigation Pressure and Rates of Cesarean Section and Vaginal Birth after Cesarean Section. Medical Care, 47, 234-242. https://doi.org/10.1097/MLR.0b013e31818475de

[35] Anim-Somuah, M., Smyth, R.M. and Jones, L. (2011) Epidural versus Nonepidural or No Analgesia in Labour. The Cochrane Database of Systematic Reviews, 19, No. CD000331. https://doi.org/10.1002/14651858.CD000331.pub3

[36] Hodnett, E.D., Gates, S., Hofmeyr, G.J. and Sakala, C. (2013) Continuous Support for Women during Childbirth. Cochrane Database of Systematic Reviews, 16, No. CD003766. https://doi.org/10.1002/14651858.CD003766.pub5

Submit or recommend next manuscript to SCIRP and we will provide best service for you:

Accepting pre-submission inquiries through Email, Facebook, LinkedIn, Twitter, etc. A wide selection of journals (inclusive of 9 subjects, more than 200 journals) Providing 24-hour high-quality service User-friendly online submission system Fair and swift peer-review system Efficient typesetting and proofreading procedure Display of the result of downloads and visits, as well as the number of cited articles Maximum dissemination of your research work

Submit your manuscript at: http://papersubmission.scirp.org/

Or contact health@scirp.org 